\title{
Thalamic mechanisms of chest pain in the absence of cardiac pathology
}

\begin{abstract}
Mechanisms to explain angina in patients with normal coronary arteries have often invoked ischaemia caused by an obscure metabolic defect or obstruction in a microscopic vascular bed. ${ }^{1}$ Recent reports of abnormal sensory mechanisms in such patients may explain some cases. On page 436 Frøbert and coworkers describe somatosensory findings in patients with angina despite normal coronary angiograms. ${ }^{2}$ In these patients oesophageal stimulation evoked a sensation like angina. Diminished evoked potentials were found in response to electrical stimulation of the oesophagus. Somatosensory thresholds to repetitive electrical oesophageal stimuli were raised although thresholds for stimulation of the skin were normal. These results suggest altered processing of visceral nociceptive signals in patients who have angina despite normal coronary angiograms. Prolonged changes in thalamic metabolism were found following angina ${ }^{3}$ in positron emission tomographic studies. Therefore, altered somatosensory function in patients with angina despite normal coronary angiograms may result from altered thalamic function. Recent studies of human thalamic physiology suggest a model which is consistent with a thalamic mechanism for some cases of angina with normal coronary angiograms.
\end{abstract}

\section{Thalamic mechanisms of acute somatic and visceral pain}

Thalamic physiology has been studied in patients undergoing stereotactic procedures for treatment of movement disorders and chronic somatic pain. ${ }^{4-7}$ These studies demonstrate that the postero-inferior aspect of the human principal sensory nucleus (Vc, ventralis caudalis) is an important structure in pain signalling pathways. The spinothalamic tract (STT) terminates in this region ${ }^{8}$; the sensation of pain can be evoked by stimulation ${ }^{5}$ and cells responding to painful stimuli are recorded in this area. ${ }^{4}$ The location of cells responding to painful stimuli correlates significantly with sites where stimulation evokes pain, demonstrating that these cells signal pain. ${ }^{45}$ These observations show that cells at the postero-inferior aspect of the Vc signal somatic pain.

Anatomical and physiological studies are also consistent with involvement of the region posterior and inferior to $\mathrm{Vc}$ in cardiac sensation. STT cells in the upper thoracic spinal cord that project to the ventralis posterior, the monkey nucleus equivalent to $\mathrm{Vc}$, respond to coronary artery occlusion. ${ }^{9}$ Additionally, cells at the posterior aspect of the nucleus equivalent to $\mathrm{Vc}$ in the cat respond to stimulation of cardiac sympathetic nerves. ${ }^{10}$ Neurons in the thalamic principal sensory nucleus also encode visceral inputs from the gastrointestinal and genitourinary systems in monkeys. ${ }^{11}$ Therefore experimental studies suggest that cells in the thalamic principal sensory nucleus encode noxious vis- ceral stimuli. Finally, this region projects to the insular cortex $^{812}$ where stimulation evokes visceral sensations in humans. ${ }^{13}$ Visceral pain can also be evoked by stimulation posterior to Vc. ${ }^{6}$ The sensation of angina was evoked by thalamic stimulation in a patient who had a history of unstable angina, which became stable after coronary angioplasty. Stimulation posterior to Vc stimulation evoked a sensation (stimulation-associated angina) that was almost identical to the patient's usual angina. Clinical, haemodynamic, electrophysiological, and biochemical measures of cardiac function showed no evidence of ischaemia at the time. In combination with experimental results, this report ${ }^{6}$ argues forcefully that the region of human Vc signals visceral sensation.

\section{Thalamic stimulation reproduces memory of chest pain}

The suggestion that stimulation-evoked angina was due to conditioning by a previous experience of the pain rests on analysis of another case of stimulation-evoked chest pain. ${ }^{7}$ In this case atypical chest pain was evoked by stimulation in the area posterior to Vc during stereotactic thalamotomy for essential tremor in a man with a diagnosis of panic disorder. The chest pain produced by microstimulation was almost identical to that occurring during his panic attacks. The operative record, cardiovascular exam, enzymes, electrocardiograms (ECGs), thallium stress test, and an ECG done postoperatively during atypical chest pain, ruled out a cardiac source of the pain.

In contrast to the results in this patient with atypical chest pain, stimulation during explorations in 100 patients who had chest pain without a strong affective dimension never evoked chest pain with an affective dimension. ${ }^{7}$ Sharp chest pain, like that in the patient with atypical chest pain, but without the affective dimension ${ }^{7}$ was evoked at three sites in other patients, showing that sharp chest pain and the affective dimension can be dissociated. Stimulation-evoked chest pain with a strong affective dimension was only evoked by stimulation postero-inferior to the $\mathrm{Vc}$ if the patient had had prior experience of pain with a strong affective dimension. In contrast, chest pain with a strong affective component was never evoked in patients without prior experience of chest pain with a strong affective dimension. ${ }^{6}$ Therefore stimulation-evoked pain and a strong affective dimension may be coupled after conditioning by a prior experience of pain with a strong affective dimension.

Thalamocortical mechanisms of pain memory Stimulation-evoked pain memory may be related to the projection from the thalamus to the cortical areas that pro- 
ject to the medial temporal lobe. The region posterior and inferior to Vc projects to the anterior insular cortex, the parietal operculum, and secondary somatosensory cortex-SII. ${ }^{812}$ There is good evidence that cortical areas receiving input from the region posterior and inferior to Vc are involved in pain processing. ${ }^{7}$ SII and insular cortical areas involved in pain processing also satisfy criteria ${ }^{14}$ for areas involved in sensory memory through corticolimbic connections. ${ }^{7}$ Thus there is anatomical and physiological evidence to support a model of conditioned limbic structures that can be triggered by innocuous and unrelated somatic or emotional stimuli to reproduce a memory of the affective and sensory aspect of pain through thalamocorticolimbic connections. Evidence for such a model is also found in the production of emotional phenomenon by stimulation of the amygdala in patients whose seizure aura included those emotional phenomena. ${ }^{15}$ These results suggest that peripheral stimuli activating these same pathways could reproduce the pain, including the affective dimension. Specifically, innocuous stimuli converging with cardiac afferents might provoke the previous experience of angina.

\section{Examples of memory for visceral pain}

The model explaining reproduction of previously experienced pain is not unique to cardiac pain. Memory of previously experienced visceral pain has been well demonstrated for renal colic. Giamberardino and coworkers showed significant sensory abnormalities in patients with previous episodes of renal colic who were currently free of renal disease. ${ }^{16}$ Almost all of these patients (8/9) had decreased pain thresholds for muscular stimuli applied to the flank on the side of a previous episode of renal colic. ${ }^{16}$ Thresholds were normal on the unaffected side. A few patients (2/9) continued to have episodes of pain "similar to that which was perceived when the calculus was still present in the renal tract". One of these patients experienced an acute attack of renal colic associated with autonomic signs in the absence of any evidence of a calculus. ${ }^{16}$ In this same patient sensory stimulation could reproduce the pain of colic as predicted above. This may be analogous to the reproduction of angina by stimulation of the oesophagus in patients who have angina despite normal coronary arteries. ${ }^{2}$

Thus anatomical and physiological studies define a thalamic model which explains memory of previously experienced pain. Pain following renal colic is an example of a condition which could be explained by this model. The same model might explain spontaneous or stimulation-evoked angina in patients who have previously experienced angina but now have angina in the absence of coronary artery disease. Patients who have angina after successful revascularisation may have pain of this type.
However, these studies raise as many questions as they answer. In particular, how would patients who are naive for ischaemia develop a memory of angina-would they have had to experience at least one episode of myocardial ischaemia, perhaps from coronary spasm? Could angina with normal coronary arteries be the result of a lowered threshold to sensory signals in cardiac afferents during exercise in combination with memory for oesophageal or other chest pain?

F A LENZ

Department of Neurosurgery, P M DOUGHERTY

Meyer Building 7-113,

fohns Hopkins Hospital,

600 North Wolfe Street,

Baltimore, MD 21287-7713,

USA

Department of Cardiology

Gohns Hopkins Hospital,

600 North Wolfe Street,

Baltimore, MD 21287-7713,

USA

Some of the studies described in this editorial were supported by grants to FAL from the Eli Lilly Corporation and the NIH (NS28598, K08 NS01384, P01 NS32386).

1 Cannon ROIII. Angina pectoris with normal coronary angiograms. Cardiology Clin 1991;9:157-66.

2 Frobert O, Arendt-Nielsen L, Bak P, Funch-Jensen P, Bagger JP. Pain perception and brain evoked potentials in patients with angina despite normal coronary angiograms. Heart 1996;75:436-41.

3 Rosen SD, Paulesu E, Frith CD, Frackowiak RSJ, Davies GJ, Jones T, et al. Central nervous pathways mediating angina pectoris. Lancet 1994; 344:147-50.

4 Lenz FA, Seike M, Lin YC, Baker FH, Rowland LH, Gracely RH, et al. Neurons in the area of human thalamic nucleus ventralis caudalis respond to painful heat stimuli. Brain Res 1993;623:235-40.

5 Lenz FA, Seike M, Lin YC, Baker FH, Richardson RT, Gracely RH. Thermal and pain sensations evoked by microstimulation in the area of the human ventrocaudal nucleus (Vc). Ұ Neurophysiol 1993;70:200-12.

6 Lenz FA, Gracely RH, Hope EJ, Baker FH, Rowland LH, Dougherty PM, et al. The sensation of angina can be evoked by stimulation of the human thalamus. Pain 1994;59:119-25.

7 Lenz FA, Gracely RH, Romanoski AJ, Hope EJ, Rowland LH, Dougherty PM. Stimulation in the human somatosensory thalamus can reproduce both the affective and sensory aspects of previously experienced pain. Nature Med 1995;1:910-3.

8 Mehler WR. The anatomy of the so-called "pain tract" in man: an analysis of the course and distribution of the ascending fibers of the fasciculus anterolateralis. In:French JD, Porter RW, eds. Basic research in paraplegia. anterolateralis. In:French $\mathrm{J}$,

9 Blair RW, Ammons WS, Foreman RD. Responses of thoracic spinothalamic and spinoreticular cells to coronary artery occlusion. $\mathcal{f}$ Neurophysiol

10 Taguchi H, Masuda T, Yokota T. Cardiac sympathetic afferent input onto neurons in nucleus ventralis posterolateralis in cat thalamus. Brain Res 1987;436:240-52.

11 Bruggemann J, Shi T, Apkarian AV. Squirrel monkey lateral thalamus. II. Viscerosomatic convergent representation of urinary bladder, colon, and esophagus. F Neurosci 1994;14:6796-814.

12 Van Buren JM, Borke RC. Variations and connections of the human thalamus. Berlin:Springer Verlag, 1972 .

13 Penfield W, Rasmussen T. The cerebral cortex of man. New York:Macmillan, 1955.

14 Mishkin M. Analogous neural models for tactual and visual learning. Neuropsychology 1979;17:139-51.

5 Halgren E, Walter RD, Cherlow DG, Crandall PH. Mental phenomena evoked by electrical stimulation of the human hippocampal formation and amygdala. Brain 1978;101:83-117.

16 Vecchiet L, Giamberardino MA, de Bigontina P. Referred pain from viscera: when the symptom persists despite the extinction of the visceral focus. Adv Pain Res Ther 1992;20:101-10. 